\title{
Effect of anterior crowding or spacing on oral health-related quality of life: a cross-sectional study
}

This article was published in the following Dove Press journal:

Patient Preference and Adherence

\author{
Ali H Hassan' \\ Nour M Hobani \\ Sara M Almokri ${ }^{3}$ \\ Nour M Almokri \\ Faiza G Alotibi ${ }^{4}$ \\ Ehab N Alshouibi ${ }^{5}$ \\ 'Department of Orthodontics, \\ King Abdulaziz University, Faculty \\ of Dentistry, Jeddah, Saudi Arabia; \\ 2Department of Periodontolgy, \\ King Abdulaziz University, Faculty \\ of Dentistry, Jeddah, Saudi Arabia; \\ ${ }^{3}$ Saudi Board of Prosthodontics, \\ King Abdulaziz University, Faculty \\ of Dentistry, Jeddah, Saudi Arabia; \\ ${ }^{4}$ Department of Orthodontics, King \\ Saud University, Riyadh, Saudi Arabia; \\ ${ }^{5}$ Department of Dental Public Health, \\ King Abdulaziz University, Faculty of \\ Dentistry, Jeddah, Saudi Arabia
}

Introduction: Oral health-related quality of life (OHRQoL) involves many aspects such as chewing ability, sleeping, social interactions, self-esteem, and satisfaction with life and oral health. The increasing research interest in OHRQoL began only after the shortcomings of previous approaches of treating symptoms only and neglecting the patient's self-perception were revealed.

Patients and methods: The current study design is a cross-sectional study of patients who attended King Abdulaziz University Dental Hospital (Jeddah, Saudi Arabia) and King Saud University Dental Hospital (Riyadh, Saudi Arabia). After obtaining their verbal consent, young adult and adult patients (mean age 25.19 \pm 7.29 years old) with anterior spacing or crowding were recruited to participate in the study. They filled the Arabic short version of the oral-health impact profile-14 questionnaire after a clinical evaluation of the severity of their spacing or crowding. Parameters of spacing/crowding severity assessment were as follows: $<4$, mild; $4-8$, moderate; and $>8$, severe. Data were analyzed using the chi-square test in SPSS statistical package. The level of significance was set to $<0.05$.

Results: The sample size of this study was 308 subjects. Findings indicated a statistically significant ( $p=0.001)$ association between anterior spacing malocclusion (ASM) with Q5 "self-consciousness", since $64.2 \%$ of patients with ASM reported being self-conscious. Results indicated a statistically significant association between educational level with anterior crowding malocclusion (ACM; $p=0.02$ ) and ASM ( $p=0.01)$ with Q3 “painful aching”. Moreover, findings indicated a statistically significant association $(p=0.04)$ between income and ACM with Q5 "self-consciousness". On the other hand, results showed no significant association between gender with either ACM or ASM.

Conclusion: This study sheds light on how anterior malocclusion (crowding or spacing) impacts OHRQoL negatively, especially heightening self-consciousness about their appearance. These effects should be addressed by the orthodontist during the course of treatment.

Keywords: OHRQoL, malocclusion, OHIP-14, patient preference

\section{Introduction}

Oral health-related quality of life (OHRQoL) is defined as "The subjective experience of symptoms related to oral conditions that have an impact on well-being." There are abundant articles discussing the effect of malocclusion generally or a specific type of malocclusion on the quality of life or self-esteem of patients. The increasing research interest in OHRQoL began only after the shortcomings of previous approaches of treating symptoms only and neglecting the patient's self-perception were revealed. ${ }^{2,3}$ Some patients with mild malocclusion may report greater concerns than those with severe forms and a different self-perception. OHRQoL involves many aspects such as chewing ability, sleeping, social interactions, self-esteem, and satisfaction with life
Department of Orthodontics, Faculty of Dentistry, King Abdulaziz University, PO Box 80209, Jeddah 21589,

Kingdom of Saudi Arabia

Tel +966503647336

Email aakbr@kau.edu.sa 
and oral health. ${ }^{3,4}$ Pelo et al conducted a study comparing two approaches for correcting skeletal discrepancies. One is the traditional approach of starting treatment with an orthodontic decompensation phase, followed by orthognathic surgery, and then orthodontic treatment again, and the other new approach is with surgery first and then orthodontic treatment. They used the oral-health impact profile-14 (OHIP-14) and the orthognathic quality of life questionnaires. They observed a significant and an immediate improvement in quality of life in the surgery-first group. One of the important factors that contributed to this improvement is the fact that the second approach corrects facial esthetics and concerns faster. ${ }^{5}$ Choi et al assessed OHRQoL in 472 patients aged $21.1 \pm 8.6$ years with OHIP-14 survey and food-intake ability (FIA) with the FIA questionnaire. They found no statistical difference between males and females in OHRQoL or masticatory ability. Moreover, most patients were seeking orthodontic treatment for esthetic purposes rather than to improve functional limitations. Participants $>30$ years old reported lower masticatory function and lower OHRQoL. Finally, they concluded that there is a linear relationship between malocclusion severity, OHRQoL score, and masticatory ability. ${ }^{6}$ Barbosa et al conducted a cross-sectional study in Brazil that involved 167 school children aged between 8 and 14 years old to evaluate the impact of malocclusion on quality of life. They concluded that malocclusion affects a child's quality of life negatively. ${ }^{7}$ Hassan et al assessed OHRQoL using the oral health impact profile (OHIP) questionnaire in correlation with the index of orthodontic treatment needs (IOTN) in 120 children between the ages of 12 and 15 years seeking orthodontic treatment and their parents. They found that as the orthodontic treatment need increased, OHRQoL decreased. ${ }^{8}$ Ramos-Jorge et al conducted a cross-sectional study in Brazil involving 451 children aged 3-5 years to assess the relationship between different types of malocclusion and quality of life. They found that anterior open bite was associated with a negative impact on the quality of life of preschool children and their families. ${ }^{9}$ Hassan and Amin assessed OHRQoL using the OHIP-14 questionnaire and the IOTN in 366 young adults aged 21-25 years seeking orthodontic treatment. They concluded that malocclusion affects OHRQoL. ${ }^{10}$ One important factor that affects quality of life is the patient's perception. It is evident that some patients with severe malocclusion report a good quality of life. Hence, they do not seek orthodontic treatment. Thiruvenkadam et al compared the quality of life of children with a history of orthodontic treatment with patients who never had orthodontic treatment using a short version of the Child Oral Health
Impact Profile (COHIP). They concluded that patients who had orthodontic treatment had a lower quality of life than those who never had orthodontic treatment. ${ }^{3}$

Anterior spacing and crowding are prevalent types of malocclusions in Saudi population. ${ }^{11,12}$ Hence, we were interested to explore the effects of these specific types of malocclusion on OHRQoL. This knowledge can help orthodontists to prioritize solving problems that are significantly affecting patient's OHRQoL as early as possible in the treatment course. In addition, no study has assessed the effect of anterior spacing or crowding specifically on the quality of life. Therefore, the current study was aimed to evaluate the OHRQoL in subjects with anterior spacing or crowding.

\section{Patients and methods}

The current study design is a cross-sectional study of patients who attended King Abdulaziz University Dental Hospital (Jeddah, Saudi Arabia) and King Saud University Dental Hospital (Riyadh, Saudi Arabia). Ethical approval (number \#0010117) was obtained from King Abdulaziz University Dental Hospital. After obtaining their verbal consent, young adult and adult patients (mean age 25.19 \pm 7.29 years old) with anterior spacing or crowding were recruited to participate in the study. Verbal consent was obtained from the parent or legal guardian of any participants who were under the age of 18 . Verbal consent was approved by ethical approval committee of King Abdulaziz University Dental Hospital. The patients filled the Arabic short version of the OHIP-14 questionnaire after a clinical evaluation of the severity of their spacing or crowding. ${ }^{13}$ Parameters of spacing/crowding severity assessment were as follows: $<4$, mild; 4-8, moderate; and $>8$, severe. ${ }^{14}$

Exclusion criteria included the following:

- Patients $<15$ years old

- Current or history of orthodontic treatment ${ }^{3}$

- Severe dentofacial anomalies, including cleft lip and palate or obvious malocclusions, such as severe unilateral or bilateral crossbite

- Patients with extraction spaces

- Patients with or in need of extensive dental treatment

- Patients with extensive anterior dental treatment

- Patients with systemic diseases

\section{Statistical analysis}

Data were analyzed using the chi-square test in SPSS statistical package (version 21; SPSS, Chicago, IL, USA). The level of significance was set to $<0.05$. The scores of OHIP-14 were dichotomized to absence (if the answer is "never" or 
"hardly ever") and presence (if the answer is "occasionally," "fairly often," and "very often") of impact on OHRQoL.

\section{Results}

The sample size of this study was 308 subjects. Descriptive analysis illustrated the sample characteristics with $53.2 \%$ males with the mean age of 25.2 years. In terms of educational level, almost $58 \%$ of the studied population held bachelor's degrees. An income of $<3,000$ SR represents the majority of the studied population (40\%) followed by those with $>9,000$ SR (23\%; Table 1). The distribution of anterior crowding malocclusion (ACM) and anterior spacing malocclusion (ASM) were divided into mild, moderate, and severe based on the severity of the cases. A trend was observed for both ACM and ASM where those with a mild form of malocclusion were more prevalent followed by moderate and severe forms. Upon comparison between crowding and spacing malocclusions, it was found that crowding was a more prevalent malocclusion with $56.5 \%$ than spacing, which represents $43.5 \%$.

Bivariate analyses (chi-square test) were performed to test potential association between ACM and ASM with each of the OHIP-14 questions. Findings indicated a statistically significant ( $p=0.001$ ) association between ASM with Q5 "selfconsciousness," since $64.2 \%$ of patients with ASM reported being self-conscious (Table 2). The potential associations between ACM and ASM with descriptive data (gender, educational level, and income) were tested using the chi-square test. Results indicated a statistically significant association between educational level with ACM $(p=0.02)$ and ASM ( $p=0.01)$ with Q3 "painful aching”. Moreover, findings indicated a statistically significant association $(p=0.04)$ between income and ACM with Q5 "self-consciousness".

Table I Sample characteristics and demographic distribution

\begin{tabular}{ll}
\hline Sample characteristics & Number (\%) \\
\hline Gender & \\
$\quad$ Male & $164(53.2)$ \\
Female & $144(46.8)$ \\
$\quad$ Total & 308 \\
Mean age \pm SD, years & $25.19 \pm 7.29$ \\
Educational level & \\
Below bachelor's degree & $113(36.7)$ \\
Bachelor's degree & $178(57.8)$ \\
Post-graduate degrees & $17(5.5)$ \\
Income, SR & \\
$<3,000$ & $123(39.9)$ \\
$3,000-6,000$ & $56(18.2)$ \\
$>6,000-9,000$ & $58(18.8)$ \\
$>9,000$ & $71(23.1)$ \\
\hline
\end{tabular}

Table 2 The potential association between anterior malocclusion and OHIP-I4

\begin{tabular}{|c|c|c|}
\hline $\begin{array}{l}\text { OHIP-I4 daily } \\
\text { activity }\end{array}$ & $\begin{array}{l}\text { Crowding } \\
\mathrm{n}(\%) \\
(\mathrm{N}=174)\end{array}$ & $\begin{array}{l}\text { Spacing } \\
n(\%) \\
(N=134)\end{array}$ \\
\hline \multicolumn{3}{|c|}{ QI-Had problems pronouncing words } \\
\hline Presence of impact & $36(20.6)$ & $47(35.1)$ \\
\hline$p$-value & $0.001 *$ & $0.001 *$ \\
\hline \multicolumn{3}{|c|}{ Q2-Felt that the sense of taste worsened } \\
\hline Presence of impact & $21(12.1)$ & $26(19.4)$ \\
\hline$p$-value & $0.001 *$ & $0.00 I^{*}$ \\
\hline \multicolumn{3}{|c|}{ Q3-Had painful aching in the mouth } \\
\hline Presence of impact & $98(56.3)$ & $78(58.2)$ \\
\hline$p$-value & 0.09 & 0.05 \\
\hline \multicolumn{3}{|c|}{ Q4-Found it uncomfortable to eat any food } \\
\hline Presence of impact & $78(45.8)$ & $63(47)$ \\
\hline$p$-value & 0.17 & 0.49 \\
\hline \multicolumn{3}{|c|}{ Q5-Had been self-conscious } \\
\hline Presence of impact & $79(45.4)$ & $86(64.2)$ \\
\hline$p$-value & 0.22 & $0.001 *$ \\
\hline \multicolumn{3}{|l|}{ Q6-Felt tense } \\
\hline Presence of impact & $66(38)$ & $67(50)$ \\
\hline$p$-value & $0.001 *$ & 1 \\
\hline \multicolumn{3}{|c|}{ Q7-Had an unsatisfactory diet } \\
\hline Presence of impact & $39(22.4)$ & $32(23.9)$ \\
\hline$p$-value & $0.00 I^{*}$ & $0.001 *$ \\
\hline \multicolumn{3}{|c|}{ Q8-Had to interrupt meals } \\
\hline Presence of impact & $38(21.8)$ & $33(24.6)$ \\
\hline$p$-value & $0.001 *$ & $0.001 *$ \\
\hline \multicolumn{3}{|c|}{ Q9-Found it difficult to relax } \\
\hline Presence of impact & $4 \mid(23.6)$ & $37(27.6)$ \\
\hline$p$-value & $0.001 *$ & $0.00 I *$ \\
\hline \multicolumn{3}{|c|}{ QI0-Had been a bit embarrassed } \\
\hline Presence of impact & $74(42.5)$ & 7I (53) \\
\hline$p$-value & $0.04 *$ & 0.49 \\
\hline \multicolumn{3}{|c|}{ QII-Had been irritable with other people } \\
\hline Presence of impact & $59(33.9)$ & $60(44.8)$ \\
\hline$p$-value & $0.001 *$ & 0.22 \\
\hline \multicolumn{3}{|c|}{ Q12-Had difficulty doing usual jobs } \\
\hline Presence of impact & $35(20.1)$ & $29(21.6)$ \\
\hline$p$-value & $0.001 *$ & $0.001 *$ \\
\hline \multicolumn{3}{|c|}{ Q13-Felt that life in general was less satisfactory } \\
\hline Presence of impact & $37(21.3)$ & $39(29.1)$ \\
\hline$p$-value & $0.001 *$ & $0.001 *$ \\
\hline \multicolumn{3}{|c|}{ QI4-Had been totally unable to function } \\
\hline Presence of impact & $20(11.5)$ & $16(11.9)$ \\
\hline$p$-value & $0.001 *$ & $0.001 *$ \\
\hline
\end{tabular}

Notes: Absence of impact $=$ never or hardly ever; presence of impact $=$ occasionally, fairly often, or very often. * $p$-value is significant.

Abbreviation: OHIP-14, oral-health impact profile-14.

On the other hand, results showed no significant association between gender with either ACM or ASM.

\section{Discussion}

This study used the Arabic version of OHIP-14, which was validated in a convenient sample of the Saudi population. ${ }^{10,13,15-18}$ This questionnaire was used in several cross-sectional and 
longitudinal studies to assess the impact of several oral-health diseases and disorders, including malocclusion. ${ }^{19,20}$

Individuals seeking dental treatment with either ACM or ASM were recruited from King Abdulaziz University Dental Hospital in Jeddah and King Saud University Dental Hospital in Riyadh. The sample size consisted of 308 subjects, $53.2 \%$ males and $46.8 \%$ females with the mean age of 25.2 years. In terms of educational level, almost $58 \%$ of the studied population held a bachelor's degree. An income of $<3,000 \mathrm{SR}$ represented the majority of the studied population $(40 \%)$. Patients with ACM constituted $56.5 \%$ of the sample compared to $43.5 \%$ of patients with ASM.

In the current study, "painful aching", "chewing efficiency", "diet choices", and "meal interruption" were not significant, supporting Daniels and Richmond findings stating that functional aspects have minimal effect on the OHRQoL among adolescents. ${ }^{21}$ On the other hand, de Oliveira and Sheiham, and Hassan and Amin reported that functional parameters were significantly affected. ${ }^{10,22}$ This may be attributed to the fact that this study assessed anterior malocclusion (crowding and spacing), which may have no or minimal impact on functional abilities. Moreover, in the present study, "self-consciousness" was significantly affected by ASM. This finding supports the results of de Oliveira, who reported that self-consciousness increased for patients with malocclusion. ${ }^{22}$

This study assessed the association between anterior malocclusion with descriptive data (gender, educational level, and income), and we found no statistically significant association with gender. This finding agrees with Hassan and Amin and disagrees with de Oliveria and Sheiham, Cicciu et al, and Ulinski et al who found that gender affected OHRQoL perception significantly; under similar conditions, females reported more discontent with their appearance than males. ${ }^{10,23-25}$ Furthermore, Tsakos et al reported that educational level affected OHRQoL negatively in elderly people. ${ }^{26}$ The current study found educational level to be a significant factor for patients with ACM and ASM only regarding one question, which was Q5 "self-consciousness".

The present study's findings agree with those of other studies that use OHIP-14, which concluded that malocclusion affects OHRQoL negatively. ${ }^{1,6,27,28}$ A limitation of the current study must be considered. Subjects were recruited from hospitals; thus, this sample might not represent the characteristics of the rest of the population who did not seek dental treatment, thereby causing an unequal distribution of malocclusion severity and age.

\section{Conclusion}

This study shed light on how anterior malocclusion (crowding or spacing) impacts OHRQoL negatively, especially heightening self-consciousness about their appearance. These effects should be addressed by the orthodontist during the course of treatment.

\section{Disclosure}

The authors report no conflicts of interest in this work.

\section{References}

1. Yamane-Takeuchi M, Ekuni D, Mizutani S, et al. Associations among oral health-related quality of life, subjective symptoms, clinical status, and self-rated oral health in Japanese university students: a crosssectional study. BMC Oral Health. 2016;16(1):127.

2. Allen PF. Assessment of oral health related quality of life. Health Qual Life Outcomes. 2003;1(1):40.

3. Asokan S, John JB, Geetha Priya PR, Prathiba J, Thiruvenkadam G. Oral health-related quality of life of children seeking orthodontic treatment based on child oral health impact profile: a cross-sectional study. Contemp Clin Dent. 2015;6(3):396-400.

4. Settineri S, Rizzo A, Liotta M, Mento C. Italian validation of the psychosocial impact of dental aesthetics questionnaire (PIDAQ). Health. 2014; 6(16):2100-2108.

5. Pelo S, Gasparini G, Garagiola U, et al. Surgery-first orthognathic approach vs traditional orthognathic approach: oral health-related quality of life assessed with 2 questionnaires. Am J Orthod Dentofac Orthop. 2017;152(2):250-254.

6. Choi SH, Kim JS, Cha JY, Hwang CJ. Effect of malocclusion severity on oral health-related quality of life and food intake ability in a Korean population. Am J Orthod Dentofacial Orthop. 2016;149(3):384-390.

7. Barbosa TS, Gavião MB, Leme MS, Castelo PM. Oral health-related quality of life in children and preadolescents with caries, malocclusions or temporomandibular disorders. Oral Health Prev Dent. 2016; 14(5):389.

8. Hassan AH, Hassan MH, Linjawi AI. Association of orthodontic treatment needs and oral health-related quality of life in Saudi children seeking orthodontic treatment. Patient Prefer Adherence. 2014;8: 1571-1579.

9. Ramos-Jorge J, Motta T, Marques LS, Paiva SM, Ramos-Jorge ML. Association between anterior open bite and impact on quality of life of preschool children. Braz Oral Res. 2015;29(1):46.

10. Hassan AH, Amin HE. Association of orthodontic treatment needs and oral health-related quality of life in young adults. Am J Orthod Dentofac Orthop. 2010;137(1):42-47.

11. AlQarni MA, Banihuwaiz AH, Alshehri FD, Alqarni AS, Alasmari DS. Evaluate the malocclusion in subjects reporting for orthodontic treatment among Saudi population in Asser Region. J Int oral Heal. 2014; 6(4):42-46.

12. Asiry MA. Occlusal Status among 12-16 year-old school children in Riyadh, Saudi Arabia. J Int oral Heal. 2015;7(5):20-23.

13. Al-Jundi MA, Szentpétery A, John MT. An Arabic version of the oral health impact profile: translation and psychometric properties. Int Dent J. 2007;57(2):84-92.

14. Mitchell L. An Introduction to Orthodontics. 4th edition. Oxford University Press. 2013.

15. Khalifa N, Allen PF, Abu-bakr NH, Abdel-Rahman ME. Psychometric properties and performance of the oral health impact profile (OHIP14s-ar) among Sudanese adults. J Oral Sci. 2013;55(2):123-132.

16. Al Habashneh R, Khader YS, Salameh S. Use of the Arabic version of oral health impact profile-14 to evaluate the impact of periodontal disease on oral health-related quality of life among Jordanian adults. J Oral Sci. 2012;54(1):113-120. 
17. Allen PF, Locker D. Do item weights matter? An assessment using the oral health impact profile. Community Dent Health. 1997;14(3): 133-138.

18. Alghamdi MA, Farsi NJ, Hassan AH. Comparison of oral health-related quality of life of patients treated by palatal expanders with patients treated by fixed orthodontic appliances. Patient Prefer Adherence. 2017; 11:699-705.

19. Slade GD. Assessing change in quality of life using the oral health impact profile. Community Dent Oral Epidemiol. 1998;26(1):52-61.

20. Slade GD. Derivation and validation of a short-form oral health impact profile. Community Dent Oral Epidemiol. 1997;25(4):284-290.

21. Daniels C, Richmond S. The Development of the Index of Complexity, Outcome and Need (ICON). J Orthod. 2000;27(2):149-162.

22. Oliveira DC, Ferreira FM, Morosini Ide A, Torres-Pereira CC, Martins Paiva S, Fraiz FC. Impact of oral health status on the oral health-related quality of life of Brazilian male incarcerated adolescents. Oral Health Prev Dent. 2015;13(5):417-425.

23. de Oliveira CM, Sheiham A. Orthodontic treatment and its impact on oral health-related quality of life in Brazilian adolescents. J Orthod. 2004; 31(1):20-27.
24. Cicciù M, Matacena G, Signorino F, Brugaletta A, Cicciù A, Bramanti E. Relationship between oral health and its impact on the quality life of Alzheimer's disease patients: a supportive care trial. Int J Clin Exp Med. 2013;6(9):766-772.

25. Ulinski KG, do Nascimento MA, Lima AM, et al. Factors related to oral health-related quality of life of independent Brazilian elderly. Int J Dent. 2013;2013:705047.

26. Tsakos G, Sheiham A, Iliffe S, et al. The impact of educational level on oral health-related quality of life in older people in London. Eur J Oral Sci. 2009;117(3):286-292.

27. Silva LF, Thomaz EB, Freitas HV, Pereira AL, Ribeiro CC, Alves CM. Impact of malocclusion on the quality of life of Brazilian adolescents: a Population-Based Study. PLoS One. 2016;11(9):e0162715.

28. Choi SH, Kim BI, Cha JY, Hwang CJ. Impact of malocclusion and common oral diseases on oral health-related quality of life in young adults. Am J Orthod Dentofacial Orthop. 2015;147(5):587-595.

\section{Publish your work in this journal}

Patient Preference and Adherence is an international, peer-reviewed, open access journal that focuses on the growing importance of patient preference and adherence throughout the therapeutic continuum. Patient satisfaction, acceptability, quality of life, compliance, persistence and their role in developing new therapeutic modalities and compounds to optimize clinical outcomes for existing disease states are major areas of interest for the journal. This journal has been accepted for indexing on PubMed Central. The manuscript management system is completely online and includes a very quick and fair peer-review system, which is all easy to use. Visit http://www. dovepress.com/testimonials.php to read real quotes from published authors.

Submit your manuscript here: http://www.dovepress.com/patient-preference-and-adherence-journal 